\title{
Note on the hodograph of non-holonomic dynamical systems
}

\author{
By B. Spali.
}

(Received 15th February, 1940. Read 2nd March, 1940.)

1. A New Form of the Equations of Motion.

Consider a non-holonomic dynamical system specified by the $N$ coordinates $q^{i}$, and kinetic energy defined by ${ }^{1}$

$$
T=\frac{1}{2} a_{m n} \dot{q}^{m} \dot{q}^{n}
$$

where $a_{m n}$ are functions of $q^{i}$ and the dot denotes differentiation with respect to the time $t$.

Suppose the non-integrable differential equations defining the non-holonomic constraints are ${ }^{2}$

$$
\Phi_{(\alpha) i} d q^{i}=0 \text { or } \Phi_{(a) i} \dot{q}^{i}=0
$$

where $\Phi_{(a)}^{i}$ are the contravariant components of the $M$ mutually orthogonal unit vectors defining the constraints. Thus ${ }^{3}$

$$
\Phi_{(\alpha) i} \Phi_{(\beta)}^{i}=\delta_{a \beta} .
$$

The dynamical system is subject to a generalised force $X_{i}$ and a reaction $Y_{i}$ due to the constraints, such that the work done $d W$ in an arbitrary displacement $d q^{i}$ is

$$
d W=\left(X_{i}+Y_{i}\right) d q^{i}
$$

while for any constrained displacement,

$$
Y_{i} d q^{i}=0 \text {. }
$$

Comparing with (1), we see that

$$
Y_{i}=\theta_{(a)} \Phi_{(a) i},
$$

$\theta_{(a)}$ being as yet arbitrary.

The equations of motion are

$$
\frac{d}{d \iota} \frac{\partial T}{\partial q^{i}}-\frac{\partial T}{\partial q^{i}}=X_{i}+Y_{i}
$$

1 The summation convention is used, the Roman indices having the range 1 to $N$.

2 Greek indices have the range 1 to $M$ and have been enclosed in a bracket to indicate that they possess no tensorial property.

${ }^{3} \delta_{a \beta}=0$ if $a \neq \beta ; \delta_{a \beta}=1$ if $a=\beta$. 
The acceleration vector $f_{i}$ is defined by

$$
f_{i}=\frac{d}{d t} \frac{\partial T}{\partial q^{i}}-\frac{\partial T}{\partial q^{i}} .
$$

From (3), (4), and (5) the equations of motion are

$$
f_{i}=X_{i}+\theta_{(a)} \Phi_{(a) i}
$$

Multiplying by $\Phi_{(\beta)}^{i}$ and using (2) we obtain

$$
\theta_{(\beta)}=\left(f_{i}-X_{i}\right) \Phi_{(\beta)}^{i} \text {. }
$$

Substituting in (6) we obtain the equations of motion

$$
f_{i}=X_{i}+\left(f_{j}-X_{j}\right) \Phi_{(a)}^{j} \Phi_{(a) i}
$$

which can be put in the form

$$
\left(f_{j}-X_{j}\right)\left(\delta_{i}^{j}-\Phi_{(a)}^{j} \Phi_{(a) i}\right)=0,
$$

where $\delta_{i}^{j}$ is the Kronecker delta.

Introducing the mixed tensor

$$
G_{i}^{j}=\delta_{i}^{j}-\Phi_{(a)}^{j} \Phi_{(a) i},
$$

we obtain the equations of motion in the form

$$
G_{\imath}^{j}\left[f_{j}-X_{j}\right]=0 .
$$

From (7) we easily see that $G_{i}^{j} \Phi_{(\alpha) j} \equiv 0$, showing that the matrix formed from the elements $G_{i}^{j}$ is of rank $N-M$. Thus in the equations of motion (8) there are really only $N-M$ independent equations, the other $M$ equations necessary to complete the set of equations being the $M$ equations of constraint (1).

\section{Hodograph.}

The generalisation of the hodograph to holonomic dynamical systems has been given by J. L. Synge, Trans. Roy. Soc., Canada, 25 (1931), 121. The purpose of this paper is to extend the results where possible to non-holonomic dynamical systems.

For geometrical interpretation consider the manifold of configurations with the kinematical line-element

$$
d s^{2}=2 T d t^{2}=a_{m n} d q^{m} d q^{n} .
$$

From (5) we easily obtain

$$
f^{i}=\ddot{q}^{i}+\left\{\begin{array}{l}
i \\
j k
\end{array}\right\} \dot{q}^{j} \dot{q}^{k}
$$


Denoting the unit vector field parallel to the lines of force and to the acceleration by $\lambda^{i}$ and $\eta^{i}$ respectively, and the intensities by $X$ and $f$ respectively, we have that the components of the force system and the acceleration are $X \lambda^{i}$ and $f \eta^{i}$ respectively.

Suppose $\phi_{(a)}$ and $\psi_{(a)}$ are the angles between the constraint vector $\Phi_{(a)}^{i}$ and the unit vectors $\lambda^{i}$ and $\eta^{i}$ respectively. Thus

$$
\cos \phi_{(a)}=\lambda^{i} \Phi_{(a) i} ; \cos \psi_{(a)}=\eta^{i} \Phi_{(a) i} .
$$

From (7), (8) and (9) we easily derive the result

$$
f^{2}\left[1-\cos \psi_{(a)} \cos \psi_{(a)}\right]=X^{2}\left[1-\cos \phi_{(a)} \cos \phi_{(a)}\right] .
$$

This is a much more complicated equation than the simple relation $f=X$ which is satisfied by holonomic systems. This is already an indication that the corresponding relations for the hodograph of non-holonomic systems will be rather complicated.

Now paragraph 3 of Synge, l.c., defining the hodograph and 4 proving the invariance of the hodograph with respect to the base point follow also in this case as they are independent of the dynamical equations of motion. On that account they will not be reproduced here.

From (16) of Synge and from (10) above, the arc element of the hodograph is modified to

$$
d \sigma=f d t=X \sqrt{\frac{1-\cos \phi_{(a)} \cos \phi_{(a)}}{1-\cos \psi_{(a)} \cos \psi_{(\alpha)}}} d t .
$$

At once we are confronted with the difficulty that $\cos \psi_{(a)}$ depending on $\eta^{i}$ involves a knowledge of the actual dynamical path of the system and thus leads to different arc elements for the hodograph corresponding to the various paths.

In addition there is no simple relation connecting the vectors $\lambda^{i}$ and $\eta^{i}$. Thus the equations derived by Synge in the remainder of his paper cannot be modified in any simple manner for the nonholonomic systems, if we desire to obtain the corresponding relations involving the force intensity and vector. Of course all the results of Synge still hold good for non-holonomic systems if we substitute $\lambda^{i}$ and $f$ for $\eta^{i}$ and $X$ respectively.

There is one exceptional case of interest, when the constraint vectors all satisfy an equation of the form

$$
\Phi_{(a) i, j}+\Phi_{(a) j, i}=k\left\{A_{j} \Phi_{(a) i}+A_{i} \Phi_{(a) j}\right\},
$$

where $k$ is an arbitrary constant and $A_{i}$ an arbitrary vector and 
$\Phi_{(a) j, k}$ are covariant derivatives. A particular case for $k=0$ is Killing's equation'

$$
\Phi_{(a) i, j}+\Phi_{(a) j, i}=0 .
$$

The equations of motion can be put in the form ${ }^{2}$

$$
\ddot{q^{i}}+\left[\left\{\left\{_{j k}^{i}\right\}+\frac{1}{2} \Phi_{(a)}^{i}\left(\Phi_{(a) j, k}+\Phi_{(a) k, j}\right)\right] \dot{q}^{j} \dot{q}^{k}=\bar{X}^{i},\right.
$$

where

$$
\bar{X}^{i}=X^{i}-\Phi_{(a)}^{i} \Phi_{(a) j} X^{j}=G_{j}^{i} X^{j} .
$$

Using the equations (11) and (1), the equations of motion take the simple form

$$
f^{i}=\bar{X}^{i},
$$

which is exactly similar to the equations of motion of holonomic systems. Thus in this case the complete theory of Synge can be immediately taken over with the only difference that instead of the force intensity $X$ we now have the magnitude of the vector $\bar{X}^{i}$.

Equation (11) has an interesting kinematical interpretation. Differentiating (1) intrinsically with respect to $t$, we obtain

$$
\Phi_{(a) i} f^{i}+\Phi_{(a) i, j} \dot{q}^{i} \dot{q}^{j}=0 .
$$

When the vectors $\Phi_{(a)}^{i}$ satisfy (11), this obviously reduces to

$$
\Phi_{(a) i} f^{i}=0 .
$$

Thus if the constraint vectors satisfy (11), then the acceleration vector (as well as the velocity vector) lies in the vector space perpendicular to the vector space determined by the constraint vectors at each point.

We notice now from (9) that $\cos \psi_{(a)}=0$ and thus the previous difficulty about the arc element does not arise now.

Note. This problem can be attacked from a different point of view. The usual meaning of parallel displacement of a vector by means of the Christoffel symbols has been employed in defining the hodograph. Equation (13) suggests that affine parallel displacement with respect to $\Gamma_{j k}^{i}$ could be used instead in the definition of the hodograph with advantage, where

$$
\Gamma_{j k}^{i}=\Gamma_{k j}^{i}=\left\{\begin{array}{c}
i \\
j k
\end{array}\right\}+\frac{1}{2} \Phi_{(a)}^{i}\left\{\Phi_{(a) j, k}+\Phi_{(a) k, j}\right\}
$$

1. P. Eisenhart, Riemannian Geometry (Princeton, 1926), 233.

2 J. L. Synge, Tensorial methods in dynamics, Univ. Toronto Studies App. Maths. Series 2 (1936). 
'This means that if $x^{i}=x^{i}(u)$ defines a curve, then the vector $\lambda^{i}$ obtained by parallel displacement along this curve is a solution of the equation

$$
\frac{d \lambda^{i}}{d u}+\Gamma_{j k}^{i} \lambda^{j} \frac{d x^{k}}{d u}=\mathbf{0}
$$

With this type of parallel displacement, we find that the hodograph is no longer invariant with respect to the base point and thus the analogy with the hodograph of elementary dynamics is lost. It can be shown that requiring the invariance of the hodograph with respect to the base point implies that the constraint vectors satisfy Killing's equation (12), which then reduces the $\Gamma_{j k}^{i}$ parallel displacement to the usual parallel displacement.

The Mathematical Institute, 16 Chambers Street, Edinburgh, 1. 